Pacific Journal of Mathematics

INTEGRATION OF COMPACT SET-VALUED FUNCTIONS 


\section{INTEGRATION OF COMPACT SET-VALUED FUNCTIONS}

\section{Zvi Artstein And John A. Burns}

A theory of integration of compact set-valued functions is provided by applying the McShane $\mathscr{P}$-integral. This integral is a Riemann-type integral and includes the Bochner, Lebesgue and other types of integrals, and by using Riemann sums it avoids deep measure theory. Thus, the $\mathscr{P}$-integral of set-valued functions contains other types of integrals such as the Hukuhara and Debreu integrals. Generalizations of known results, including the convexity of the integral, are obtained, and the techniques do not require measure theory. Further, if a set-valued function is $\mathscr{P}$-integrable, then its integral equals the Aumann integral, where the latter is defined as the collection of integrals of selections.

1. Introduction. Considerations of summation and integration of set-valued functions go back to Minkowski. Recently, the calculus of set-valued functions was found to be very applicable in several mathematical fields, especially in control theory, mathematical economics, and statistics. Accordingly, many recent papers deal with the basic theory of integration of set-valued functions, and several approaches were established. One approach, due to Hukuhara [7], is to consider formal Riemann integration into the space of convex-compact sets. The Lebesgue integral is obtained by taking appropriate limits. A second approach was employed by Debreu [6], who used an embedding of the convex-compact sets into a Banach space and then considered the Bochner integral into this space. Yet another approach was developed by Aumann [3], which considered integration of selections of the set-valued functions. The Aumann integral is well suited for applications to various mathematical fields, and we shall give below the precise definition of this integral. In the three approaches outlined above, the main tools are measure theoretic techniques. Also, because of the usefulness of the Aumann integral, the relationships between the three types of integrals were investigated in [4], [5], and [6].

McShane [8] gave a definition of a Riemann type integral, which includes many former integrals such as the Lebesgue and Bochner integrals. Moreover, the definition has two very nice properties. First of all, the definition of McShane's integral (called the $\mathscr{P}$-integral) does not require measure theoretic concepts and is defined as a limit of Riemann-type sums. The second advantage of the McShane $\mathscr{P}$-integral is that it is defined for functions that take values in semi-groups, and 
there is no need to assume linearity of the range space. The main purpose of the present paper is to apply the McShane $\mathscr{P}_{\text {-integral to }}$ set-valued functions. We shall deal with functions defined on the real line with values in the set of compact subsets of $R^{q}$. With the Hausdorff metric on the compact subsets of $R^{q}$, the $\mathscr{P}_{\text {-integral will include the }}$ Debreu and Hukuhara integrals. (The latter are defined only for convex-compact valued functions.) Moreover, we obtain many properties of the integral without using measure theory. Thus, it is not necessary to consider an exceedingly elaborate measure theory to obtain a powerful integral, and even though measure theory is elegant, it sometimes fails to supply the simplest and most revealing proofs.

The main results are stated below. Some notations and preliminaries will be given in $\$ 2$ and the proofs are given in $\S \S 3-6$.

The first result, Theorem $\mathrm{A}$, is similar to the first theorem in Aumann [1], but its proof does not require any measure theory.- The space of compact subsets of $R^{q}$ is denoted by $\mathscr{C}\left(R^{q}\right)$.

Theorem A. If $F:[a, b] \rightarrow \mathscr{C}\left(R^{q}\right)$ is $\mathscr{P}$-integrable, then its $\mathscr{P}$ integral is a convex set.

The following result is similar to Aumann's third theorem.

THEOREM B. The function $F:[a, b] \rightarrow \mathscr{C}\left(R^{q}\right)$ is $\mathscr{P}$-integrable if and only if the set-valued function $\mathrm{coF}$, defined by $\operatorname{coF}(t)$ equals the convex hull of $F(t)$, is $\mathscr{P}$-integrable. Moreover, $(\mathscr{P}) \int_{a}^{b} F$ and $(\mathscr{P}) \int_{a}^{b} \operatorname{coF}$ are equal.

For vector-valued functions the $\mathscr{P}$-integral is equivalent to the Lebesgue integral, and hence there are the obvious measure theoretic characterizations of the $\mathscr{P}$-integral. The following result provides a characterization of $\mathscr{P}$-integrable set-valued functions and indicates the connection between $\mathscr{P}$-integrability and measurability of set-valued functions. We say that a set-valued function $F$ is measurable if for each closed set $C$ in $R^{q}$, the set $\{t: F(t) \cap C \neq \varnothing\}$ is a Lebesgue measurable subset of $[a, b]$. A set-valued function $F$ is said to be Integrably bounded if there exists a $\mathscr{P}$-integrable real-valued function $g$ such that $x \in F(t)$ implies $\|x\| \leqq g(t)$.

THEOREM C. The function $F:[a, b] \rightarrow \mathscr{C}\left(R^{q}\right)$ is $\mathscr{P}$-integrable if and only if it integrably bounded and coF is measurable.

In [2] it was shown that for convex and compact valued functions the same conditions characterize the Debreu integral. 
If $F$ is a set-valued function, then a selection of $F$ is a vectorvalued function $f:[a, b] \rightarrow R^{q}$ such that $f$ is Lebesgue measurable and $f(t) \in F(t)$ almost everywhere in $[a, b]$. The Aumann integral of $F$, denoted by $(A) \int_{a}^{b} F$, is the set of all vectors of the form $\int_{a}^{b} f$, where $f$ is a Lebesgue integrable selection of $F$. It is to be noted that the Aumann integral is defined for every set-valued function, and it may be empty. Since for scalar-valued functions the Lebesgue integral and the $\mathscr{P}$ integral are equal (see \$2), it follows that the Aumann integral is also given by

$$
(A) \int_{a}^{b} F=\left\{(\mathscr{P}) \int_{a}^{b} f: f \quad \text { is } \quad \mathscr{P} \text {-integrable and } f(t) \in F(t)\right\} .
$$

For the case where $F$ is a convex and compact set-valued function, it was shown in references [5] and [6] that the Hukuhara and the Debreu integrals equal the Aumann integral. We have the following similar result for the $\mathscr{P}$-integral, and again the proof does not use measure theoretic techniques.

ThEOREM D. If $F:[a, b] \rightarrow \mathscr{C}\left(R^{a}\right)$ is $\mathscr{P}_{\text {-integrable, then }}$ $(\mathscr{P}) \int_{a}^{b} F=(A) \int_{a}^{b} F$

The Aumann integral exists for any compact set-valued function

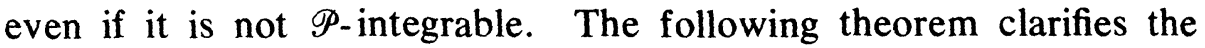
relationship between the two. It is also similar to a known result for a real-valued function, namely that if $g(t)$ is an integrably bounded real-valued function, then there exists an integrable function $f_{0}(t)$ such that $f_{0}(t) \leqq g(t)$ almost everywhere, and if $f$ is any integrable function satisfying $f(t) \leqq g(t)$ a.e., then $f(t) \leqq f_{0}(t)$ a.e.

THEOREM E. For every integrably bounded $F:[a, b] \rightarrow \mathscr{C}\left(R^{a}\right)$ such that $(A) \int_{a}^{b} F \neq \varnothing$, there is a $\mathscr{P}$-integrable function $G:[a, b] \rightarrow \mathscr{C}\left(R^{a}\right)$ such that $G(t) \subseteq F(t)$ a.e., and $G$ is maximal with respect to this property. Moreover the $\mathscr{P}$-integral of $G$ is equal to the Aumann integral of $F$. If $(A) \int_{a}^{b} F=\varnothing$, then there is no $\mathscr{P}$-integrable $G$ such that $G(t) \subseteq F(t)$ for every $t$.

2. Notations and preliminaries. Let $A$ and $B$ be subsets of $R^{q}$ and $\lambda \in R$. The sum of $A$ and $B$ is the set $A+B$ given by $A+B=\{a+b: a \in A$ and $b \in B\}$, and $\lambda A$ is the set defined by 
$\lambda A=\{\lambda a: a \in A\}$. The Hausdorff distance between $A$ and $B$ is defined by

$$
h(A, B)=\inf \{\lambda>0: A \subseteq B+\lambda U \text { and } B \subseteq A+\lambda U\}
$$

where $U$ is the closed unit ball in $R^{q}$. The set of all non-empty compact subsets of $R^{q}$, with the Hausdorff distance $h$, is a complete metric space which will be denoted by $\mathscr{C}\left(R^{q}\right)$. The convex hull of a set $A$ will be denoted by coA, and $p \cdot x$ will represent the scalar product of $p \in R^{q}$ and $x \in R^{q}$. For $A \in \mathscr{C}\left(R^{q}\right)$, the "norm" of $A$ is defined by $\|A\|=$ $h(A,\{0\})$. It is easy to verify that $\mathscr{C}\left(R^{q}\right)$ is a topological semi-group and has many interesting properties. However, we shall only be concerned with the properties needed in the context of this paper.

The following definitions are found in greater generality in McShane's memoir [8]. However, the "simple" $\mathscr{P}$-integral is equivalent to the Lebesgue integral for a vector-valued function defined on an interval. (See Theorem 13.6 and the remarks in $\$ 14$ of reference [8].]

Let $a<b$ be real numbers. A finite collection $\Pi=$ $\left\{\left(t_{1}, A_{1}\right),\left(t_{2}, A_{2}\right), \cdots,\left(t_{n}, A_{n}\right)\right\}$ is said to be a partition of $(a, b]$ if each $t_{j} \in[a, b]$, each $A_{j}$ is either empty or an interval of the form $\left(a_{j}, b_{j}\right]$ with $a \leqq a_{j}<b_{j} \leqq b$, and each point of $(a, b]$ belongs to exactly one of the sets $A_{j}$. The length of $A_{j}$ is given by $\Delta A_{j}=b_{j}-a_{j}$ if $A_{j} \neq \varnothing$ and $\Delta \varnothing=0$. A gauge on $[a, b]$ is a real-valued function $\delta:[a, b] \rightarrow(0,+\infty)$. There is no requirement that $\delta$ be continuous or bounded away from zero. Given a gauge $\delta$, a partition $\Pi$ is said to be $\delta$-fine if $A_{j} \subseteq$ $\left(t_{j}-\delta\left(t_{j}\right), t_{j}+\delta\left(t_{j}\right)\right)$ for each $j=1,2, \cdots, n$.

Digressing for a moment, it is not at all obvious that for a given gauge $\delta$ there exists any $\delta$-fine partitions of $(a, b]$. However, this may be shown to be true by an indirect proof using only the completeness of the real line. Also, it should be noted that only slight modifications are needed to extend the definitions to include infinite intervals. This will not be pursued in the present paper.

If $f$ is a function defined on $[a, b]$ with values in $R\left(\operatorname{resp} . \mathscr{C}\left(R^{a}\right)\right.$ ) and $\Pi$ is a partition of $(a, b]$, then the Riemann sum corresponding to $f$ and $\Pi$ is given by

$$
S(f ; \Pi)=\sum_{j=1}^{n} f\left(t_{j}\right) \Delta A_{j} .
$$

Such a function $f$ is said to be $\mathscr{P}$-integrable over $[a, b]$ if there exists an element $I \in R\left(I \in \mathscr{C}\left(R^{a}\right)\right)$ with the property that for each $\epsilon>0$, there exists a gauge $\delta$ such that if $\Pi$ is any $\delta$-fine partition of $(a, b]$, then

$$
\|S(f ; \Pi)-I\|<\epsilon \quad(h(S(f ; \Pi), I)<\epsilon) .
$$

The element $I$ is said to be the $\mathscr{P}$-integral of $f$ and we write $(\mathscr{P}) \int_{a}^{b} f=I$. 
At this point, we note that it is possible to derive the basic properties of the $\mathscr{P}$-integral directly from its definition, without mention of measure theory. However, since a primary objective of this paper is to compare the $\mathscr{P}$-integral and the Aumann integral of compact setvalued functions, we shall make double use of the equivalence of the $\mathscr{P}$-integral and the Lebesgue integral for vector-valued functions. That

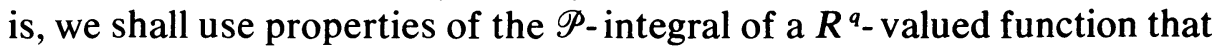
are well known for the equivalent Lebesgue integral. In particular, we use the fact that a $\mathscr{P}$-integrable function must be Lebesgue measurable and conversely that a Lebesgue integrable function is $\mathscr{P}$-integrable.

3. Proofs of Theorem $A$ and $B$. We shall need the following lemma which is due to L. Shapley and J. H. Folkman. A proof can be found in [1, Theorem 9. page 396]. It is to be noted that the proof is of combinatorial type and uses only simple properties of finite dimensional spaces.

LEMмA 3.1. (Shapley-Folkman). If $A_{1}, \cdots, A_{k}$ is a finite family of sets in $R^{a}$ such that $\left\|A_{j}\right\| \leqq L$ for a fixed $L$ and for each $j=1,2, \cdots, K$, then

$$
h\left(\sum_{j=1}^{K} A_{j}, \operatorname{co} \sum_{j=1}^{K} A_{j}\right) \leqq \sqrt{q} L
$$

Proceeding to the proof of Theorem A we assume that $F:[a, b] \rightarrow \mathscr{C}\left(R^{q}\right)$ is $\mathscr{P}$-integrable. For a given $\epsilon>0$ let $\delta$ be the gauge associated with $\epsilon$. Define $\delta^{\prime}$ by

$$
\delta^{\prime}(t)=\min \left\{\delta(t), \epsilon(\sqrt{q}(1+\|F(t)\|))^{-1}\right\}
$$

Note that by this choice, if $\Pi$ is a $\delta^{\prime}$-fine partition, then every element $F\left(t_{j}\right) \Delta A_{j}$ in the sum $S(F ; \Pi)=\sum_{j=1}^{K} F\left(t_{j}\right) \Delta A_{j}$ has norm less than $\epsilon / \sqrt{q}$ and therefore Lemma 3.1 implies that

$$
h(S(F ; \Pi), \operatorname{coS}(F ; \Pi)) \leqq \epsilon .
$$

Since a $\delta^{\prime}$-fine parition is also $\delta$-fine partition we know that $h(S(F ; \Pi)$, $\left.(\mathscr{P}) \int_{a}^{b} F\right) \leqq \epsilon$ and the latter together with the displayed inequality imply that the Hausdorff distance between $(\mathscr{P}) \int_{a}^{b} F$ and the convex set $\cos (F ; \Pi)$ is less than $2 \epsilon$. Therefore $(\mathscr{P}) \int_{a}^{b} F$ is the limit in the 
Hausdorff metric of convex sets and hence is convex. This proves Theorem A.

In order to verify Theorem B notice that since the co operation is linear, and in particular $\operatorname{coS}(F ; \Pi)=S(\operatorname{coF} ; \Pi)$, we have shown that coF is $\mathscr{P}$-integrable and $(\mathscr{P}) \int_{a}^{b} F=(\mathscr{P}) \int_{a}^{b}$ coF. Moreover the inequality, $h(S(F ; \Pi), \operatorname{coS}(F ; \Pi)) \leqq \epsilon$ in our proof, did not depend on the integrability of $F$. Therefore the reverse implication may be proven by exactly the same method and this completes the proof of Theorem B.

4. The Proof of Theorem $C$. For a set $A$ in $R^{q}$ and a vector $p$ in $R^{q},\left(R^{q}\right.$ equals the dual of $\left.R^{q}\right)$ we define $s(p, A)$ by $s(p, A)=$ $\sup \{p \cdot x: x \in A\}$. The function $s(\cdot, A)$ is known as the support function of $A$. It is a convex and positively homogeneous function and clearly real-valued if $A$ is compact. If $F$ is a set-valued function we shall use for simplicity $s(p, t)$ instead of $s(p, F(t))$. The following properties are easy to verify.

(i) $s(p, A+B)=s(p, A)+s(p, B)$.

(ii) If $\|p\| \leqq 1$, then $|s(p, A)-s(p, B)| \leqq h(A, B)$.

(iii) If $A$ and $B$ are convex, then

$$
h(A, B)=\sup _{\|p\|=1}|s(p, A)-s(p, B)| .
$$

(iv) If $p_{1}, p_{2}, \cdots$ is a dense sequence in $R^{q}$ and $A$ is compact, then

$$
\operatorname{coA}=\bigcap_{j=1}^{\infty}\left\{x: p_{i} \cdot x \leqq s\left(p_{i}, A\right)\right\}
$$

Now suppose that $F$ is $\mathscr{P}$-integrable. Properties (i) and (ii) directly

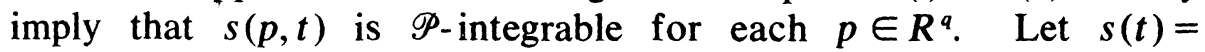
$\sum_{j=1}^{2 q}\left|s\left(e_{j}, t\right)\right|$, where $e_{1}, e_{2}, \cdots, e_{2 q}$ are the $2 q$ vectors $(0, \cdots, 0, \pm$

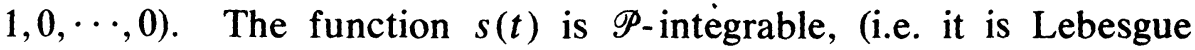
integrable) and the definition of the support function implies that $\|F(t)\| \leqq s(t)$. Thus $F$ is integrably bounded. If $p_{1}, p_{2}, \cdots$ is a dense sequence in $R^{q}$, then property (iv) implies that $\operatorname{coF}(t)=$ $\bigcap_{j=1}^{\infty}\left\{x: p_{j} \cdot x \leqq s\left(p_{j}, t\right)\right\}$. The measurability of $s\left(p_{j}, t\right)$ implies that the set-valued function $G_{t}(t)=\left\{x: p_{j} \cdot x \leqq s\left(p_{j}, t\right)\right\}$ is measurable. By Rockafellar [9; Corollary 1.3] it follows that $\operatorname{coF}(t)=\bigcap_{j=1}^{\infty} G_{j}(t)$ is measurable, and this completes the proof of the "only if" part of Theorem C.

Suppose that coF is measurable and $F$ is integrably bounded. Then for each $p$ the support function $s(p, t)$ is integrably bounded. Also, 
since $s(p, A)=s(p$, coA $)$ for any set $A$, we have that $s(p, t)$ is measurable. To see this, notice that for each $\alpha$ the set

$$
\{t: s(p, t) \geqq \alpha\}=\{t: \operatorname{coF}(t) \cap\{x: p \cdot x \geqq \alpha\} \neq \varnothing\}
$$

is a Lebesgue measurable subset of $[a, b]$.

Therefore, it follows that $s(p, t)$ is Lebesgue integrable, or equivalently, $\mathscr{P}$-integrable. Let $p_{1}, p_{2}, \cdots$ be a dense sequence in the unit sphere of $R^{q}$, and suppose $\epsilon>0$ is given. Let $\delta_{j}, j=1,2, \cdots$, be the gauge that established the $(\epsilon / 4)$-approximation of the $\mathscr{P}$-integral of $s\left(p_{i}, t\right)$. Since $F$, and hence $c o F$, is integrably bounded, there is a gauge $\delta$ and a compact set $B$ such that if $\Pi$ is any $\delta$-fine partition, then $S(\mathrm{coF} ; \Pi) \subseteq B$. Moreover, it is easy to verify that the family of support functions $\{s(\cdot, K): K$ is a compact subset $B\}$ is equicontinuous. Let $\gamma>0$ be such that if $K$ is a compact subset of $B$ and $\|p-q\|<\gamma$, then $|s(p, K)-s(q, K)|<\epsilon / 4$. Since the unit sphere is compact, there exists a finite collection, say $p_{1}, p_{2}, \cdots, p_{N(\gamma)}$, such that if $\|p\|=1$, then $\left\|p-p_{K}\right\|<\gamma$ for some $K, 1 \leqq K \leqq N(\gamma)$.

Let $\delta_{\epsilon}=\min \left\{\delta, \delta_{1}, \cdots, \delta_{N(\gamma)}\right\}$, and suppose that $\Pi_{1}$ and $\Pi_{2}$ are any two $\delta_{\epsilon}$-fine partitions. Note that $S_{1}=S\left(\mathrm{coF} ; \Pi_{1}\right)$ and $S_{2}=S\left(\mathrm{coF} ; \Pi_{2}\right)$ are compact subsets of $B$, and hence for each $p$ in the unit sphere we have that

$$
\begin{aligned}
\left|s\left(p, S_{1}\right)-s\left(p, S_{2}\right)\right| & \leqq \\
& +\left|s\left(p, S_{1}\right)-s\left(p_{K}, S_{1}\right)\right| \\
& +\left|s\left(p_{K}, S_{2}\right)-s\left(p, S_{2}\right)\right| \\
& \leqq \\
&
\end{aligned}
$$

But, $s(p, S(\mathrm{coF} ; \Pi))=S(s(p, \cdot) ; \Pi)$, and hence it follows that $\mid s\left(p, S_{1}\right)-$ $s\left(p, S_{2}\right) \mid<\epsilon$. Property (iii) implies that $h\left(S\left(\mathrm{coF} ; \Pi_{1}\right), S\left(\mathrm{coF} ; \Pi_{2}\right)\right)<\epsilon$, and we can conclude that the net of Riemann sums of coF over $\delta_{\epsilon}$-fine partitions is a Cauchy net. It is clear that this net converges to $(\mathscr{P}) \int_{a}^{b}$ coF and by Theorem B we have that $F$ is $\mathscr{P}$-integrable.

Remark 4.1. Notice that directly from the basic definitions it follows that

$$
(\mathscr{P}) \int_{a}^{b} s(p, F(t)) d t=s\left(p,(\mathscr{P}) \int_{a}^{b} F\right)
$$

This equality is a useful tool in the study of integrals of set-valued functions. 
5. The Proof of Theorem $D$. Recall that if $F$ is a set-valued function then the Aumann integral of $F$ is given by

$$
\text { (A) } \int_{a}^{b} F=\left\{(\mathscr{P}) \int_{a}^{b} f: f(t) \in F(t)\right\} \text {. }
$$

We first show that if $F:[a, b] \rightarrow \mathscr{C}\left(R^{a}\right)$ is $\mathscr{P}$-integrable then (A) $\int_{a}^{b} F \subset(\mathscr{P}) \int_{a}^{b} F$. Let $f$ be a $\mathscr{P}$-integrable selection of $F$. For a given $\epsilon>0$ let $\delta_{1}$ and $\delta_{2}$ be gauge functions that establish the $\epsilon$-approximation of the $\mathscr{P}$-integrals of $f$ and $F$ respectively. Let $\delta=\min \left(\delta_{1}, \delta_{2}\right)$. If $\Pi$ is a $\delta$-fine partition then $S(f ; \Pi)$ is an element of $S(F ; \Pi)$. Since the sums are $\epsilon$-approximations of the respective $\mathscr{P}$-integrals and since $\epsilon$ is arbitrarily small it follows that

$$
(\mathscr{P}) \int_{a}^{b} f \text { belongs to }(\mathscr{P}) \int_{a}^{b} F \text {. }
$$

We now show that the Aumann integral of $F$ contains the $\mathscr{P}$ integral of $F$. Recall the $e$ is an exposed point of the compact set $A$ if $e$ is the only point in the intersection of $A$ and a certain support hyperplane of $A$. In terms of the support function the point $e$ is an exposed point of $A$ if there exists a $p \in R^{q}$ such that

$$
p \cdot e=s(p, A) \text { and } p \cdot x<s(p, A) \text { if } e \neq x \in A \text {. }
$$

The exposed points are dense in the extreme points of a set $A$. We shall show that if $e$ is an exposed point of $(\mathscr{P}) \int_{a}^{b} F$ then $e$ belongs to the Aumann integral of $F$. Since the latter is convex and compact (Aumann [3, Theorems 1,2]) it follows that the closure of the convex hull of the exposed points of $(\mathscr{P}) \int_{a}^{b} F$, which is $(\mathscr{P}) \int_{a}^{b} F$ itself, belongs to the Aumann integral of $F$.

Let $e$ be an exposed point of $(\mathscr{P}) \int_{a}^{b} F$. Let $p$ be such that $e \neq x \in(\mathscr{P}) \int_{a}^{b} F$ implies that $p \cdot x<p \cdot e$. For every set $A$ in $R^{a}$ denote $A_{p}=\{x \in A: p \cdot x=s(p, A)\}$. The operator $A \rightarrow A_{p}$ is linear, i.e., $A_{p}+B_{p}=(A+B)_{p}$. We shall prove that $F_{p}(t)$ is $\mathscr{P}_{\text {-integrable and that }}$

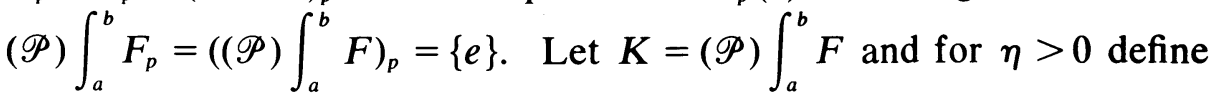
$K_{\eta}=\{x \in K: p \cdot x \geqq s(p, K)-\eta\}$. In particular we have that $K_{0}=\{e\}$. Since $e$ is the unique point in $K_{0}$ it follows that the diameter of $K_{\eta}$ tends 
to zero when $\eta$ goes to zero. Let $\epsilon>0$ be fixed. Choose $\eta$ so small that the diameter of $K_{2 \eta}$ will be less than $\epsilon$. Let $\delta$ be a gauge function for $F$ associated with $\eta$, and suppose $\Pi$ is a $\delta$-fine partition. Since

$$
h\left(S(F ; \Pi),(\mathscr{P}) \int_{a}^{b} F\right) \leqq \eta
$$

it follows that $S(F ; \Pi) \cap\{x: p \cdot x \geqq(p \cdot e)-\eta\} \neq \varnothing$. Therefore,

$$
S(F ; \Pi)_{p}=S\left(F_{p} ; \Pi\right) \subset\{x: p \cdot x \geqq(p \cdot e)-\eta\} .
$$

On the other hand, (5.1) implies that

$$
S(F ; \Pi)_{p} \subseteq\left\{x: \text { there exists } y \in K_{2 \eta} \text { such that }\|y-x\| \leqq \eta\right\},
$$

and hence $S(F ; \Pi)_{p}$ is included within an $\epsilon+\eta$ neighborhood of $\{e\}$. This completes the proof of the equality

$$
(\mathscr{P}) \int_{a}^{b} F_{p}=\left((\mathscr{P}) \int_{a}^{b} F\right)_{p}
$$

Since $F_{p}$ has at most dimension $n-1$ we can use an induction argument in order to show that the Aumann integral of $F_{p}$ equals $\{e\}$. The first step of the induction, i.e. for $n=0$, is obvious.

REMARK. We showed that $(\mathscr{P}) \int_{a}^{b} F_{p}=\left((\mathscr{P}) \int_{a}^{b} F\right)_{p}$ if $p$ determines a hyperplane which supports at an exposed point. The proof does not use measure theoretic arguments. For the Aumann integral of measurable set-valued functions it is easy to verify the same equality for every p. Thus Theorems $\mathrm{C}$ and $\mathrm{D}$ imply that $(\mathscr{P}) \int F_{p}=\left((\mathscr{P}) \int F\right)_{p}$ for every $p$. We do not have a simple proof for this which will not use measure theory.

6. Proof of Theorem $E$. Let $F$ be an integrably bounded setvalued function. Denoted by $\mathscr{F}$ the collection of equivalence classes of Lebesgue measurable selections of $F$. If $\mathscr{F}$ is empty then Theorem D implies that no integrable subfunction $G$ of $F$ exists. This proves the second statement of the theorem. Suppose now that $\mathscr{F}$ is not empty. For a vector $p$ in $R^{a}$ denote by $\rho(p, t)$ the supremum of the functions $p \cdot f(t)$, i.e. the smallest measurable function such that if $f \in F$ then $p \cdot f(t) \leqq \rho(p, t)$ for almsot every $t$. The function $\rho(p, t)$ is defined up to a set of measure zero. If $H(p, t)=\{x: p \cdot x \leqq \rho(p, t)\}$, then $H(p, t)$ is 
measurable. Let $p_{1}, p_{2} \cdots$ be a dense set in $R^{q}$. Define $G(t):[a, b] \rightarrow \mathscr{C}\left(R^{q}\right)$ by

$$
G(t)=\bigcap_{j=1}^{\infty}\left(H\left(p_{j}, t\right) \cap F(t)\right) .
$$

obviously, $G(t) \subset F(t)$. Moreover, if $p \in R^{q}$ and $f \in \mathscr{F}$, then $f$ is also a selection of $F(t) \cap H(p, t)$. Consequently, if $f \in \mathscr{F}$ then $f$ is a selection of $G$, and hence the Aumann integral of $F$ equals that of $G$. In order to show that $G$ is $\mathscr{P}$-integrable notice that $s(p, G(t))=\rho(p, t)$. This follows from the fact that $\mathscr{F}$ is also the collection of selections of $G$. Therefore,

$$
\operatorname{coG}(t)=\bigcap_{j=1}^{\infty} H\left(p_{j}, t\right)
$$

and it is measurable as a denumerable intersection of measurable functions (see Rockafeller [9, Corollary 1.3]). Remarks 4.1 and Theorem D imply that $G$ is maximal and this completes the proof.

7. P-integration into the Semigroup of all Bounded Sets. As was noted in Section 1 the $\mathscr{P}$-integral is defined for functions which might take values in general topological semigroups. In particular, we may consider the semigroup of all bounded sets with the Hausdorff semimetric. Obviously, such a set-valued function will be $\mathscr{P}$-integrable if and only if the pointwise closure of it will be $\mathscr{P}$-integrable and they will

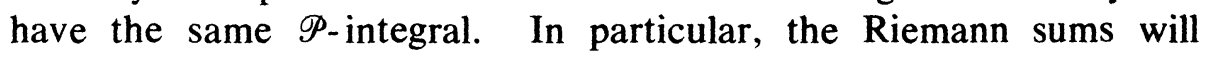
converge in the Hausdorff semi-metric. Although the Aumann integral is defined for all set-valued functions, Theorem $\mathrm{D}$ cannot be generalized to the case where compactness is not assumed. Indeed, one can easily construct a set-valued function $F(t)$ such that the closure of $F(t)$ will be constant, but there is no measurable selection of $F$.

\section{REFERENCES}

1. K. J. Arrow, and F. H. Hahn, General Competitive Analysis, Holden-Day Inc., San Francisco, 1971.

2. Z. Artstein, On the calculus of set-valued functions, Indiana Univ. Math. J., 24 (1974), 433-441.

3. R. J. Aumann, Integrals of set-valued functions, J. Math. Anal. Appl., 12 (1965), 1-12.

4. F. S. DeBlasi, and A. Lasota, Daniall method in the theory of Aumann-Hukuhara Integral of set-valued functions Atti Acad. Naz. Lincei Rendiconti Ser 8, 45 (1968), 252-256.

5. Characterization of the integral of set-valued functions, Atti Acad. Naz. Lincei Rendicoyi Ser. 8, 46 (1969), 154-157.

6. G. Debreu, Integration of correspondences, Proc. Fifth Berkeley Symp. Math. Stat. and Prob., Vol. II, Univ. Calif. Press, (1967), 351-372. 
7. M. Hukuhara, Integration des applications measurables dont la valeures compact convexe, Funk. Ekvac., 10 (1967), 205-223.

8. E. J. McShane, A Riemann-Type integral that includes Lebesgue-Stieltjes, Bochner and stochastic integrals, Memoirs Amer. Math. Soc., 88 (1969).

9. T. Rockafellar, Measurable dependence of convex sets and functions on parameters, J. Math. Anal. Appl., 28 (1969), 4-25.

Received February 25, 1974. The first author's research was partially supported by the National Science Foundation under grant GP 28931X2, and by the Office of Naval Research under grant NONR N00014-67-A-0191-0009. The second author's research was partially supported by the National Science Foundation under grant GP-2891X2.

BROWN UNIVERSITY 



\section{PACIFIC JOURNAL OF MATHEMATICS}

\section{EDITORS}

RICHARD ARENS (Managing Editor)

University of California

Los Angeles, California 90024

\section{J. DugundJI}

Department of Mathematics University of Southern California Los Angeles, California 90007

D. Gilbarg and J. Milgram

Stanford University

Stanford, California 94305

\section{ASSOCIATE EDITORS}
E. F. BECKENBACH
B. H. NeumanN
F. WoLF
K. YoshiDA

\section{SUPPORTING INSTITUTIONS}

\author{
UNIVERSITY OF BRITISH COLUMBIA \\ CALIFORNIA INSTITUTE OF TECHNOLOGY \\ UNIVERSITY OF CALIFORNIA \\ MONTANA STATE UNIVERSITY \\ UNIVERSITY OF NEVADA \\ NEW MEXICO STATE UNIVERSITY \\ OREGON STATE UNIVERSITY \\ UNIVERSITY OF OREGON \\ OSAKA UNIVERSITY
}

\author{
UNIVERSITY OF SOUTHERN CALIFORNIA \\ STANFORD UNIVERSITY \\ UNIVERSITY OF TOKYO \\ UNIVERSITY OF UTAH \\ WASHINGTON STATE UNIVERSITY \\ UNIVERSITY OF WASHINGTON \\ AMERICAN MATHEMATICAL SOCIETY
}

The Supporting Institutions listed above contribute to the cost of publication of this Journal, but they are not owners or publishers and have no responsibility for its contents or policies.

Mathematical papers intended for publication in the Pacific Journal of Mathematics should be in typed form or offset-reproduced (not dittoed), double spaced with large margins. Underline Greek letters in red, German in green, and script in blue. The first paragraph or two must be capable of being used separately as a synopsis of the entire paper. Items of the bibliography should not be cited there unless absolutely necessary, in which case they must be identified by author and Journal, rather than by item number. Manuscripts, in duplicate, may be sent to any one of the four editors. Please classify according to the scheme of Math. Reviews, Index to Vol. 39. All other communications should be addressed to the managing editor, or Elaine Barth, University of California, Los Angeles, California, 90024.

100 reprints are provided free for each article, only if page charges have been substantially paid. Additional copies may be obtained at cost in multiples of 50 .

The Pacific Journal of Mathematics is issued monthly as of January 1966. Regular subscription rate: $\$ 72.00$ a year (6 Vols., 12 issues). Special rate: $\$ 36.00$ a year to individual members of supporting institutions.

Subscriptions, orders for back numbers, and changes of address should be sent to Pacific Journal of Mathematics, 103 Highland Boulevard, Berkeley, California, 94708.

PUBLISHED BY PACIFIC JOURNAL OF MATHEMATICS, A NON-PROFIT CORPORATION Printed at Jerusalem Academic Press, POB 2390, Jerusalem, Israel.

$$
\begin{gathered}
\text { Copyright (C) } 1975 \text { Pacific Journal of Mathematics } \\
\text { All Rights Reserved }
\end{gathered}
$$




\section{Pacific Journal of Mathematics}

\section{Vol. 58, No. $2 \quad$ April, 1975}

Zvi Artstein and John Allen Burns, Integration of compact set-valued functions . . . . . . . . . 297

Mark Benard, Characters and Schur indices of the unitary reflection group $[321]^{3} \ldots \ldots \ldots . .309$

Simeon M. Berman, A new characterization of characteristic functions of absolutely continuous

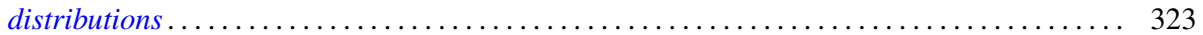

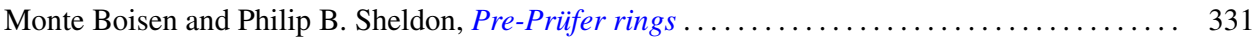

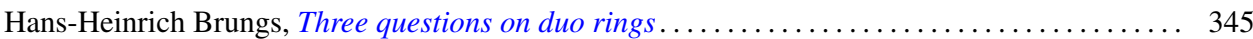

Iracema M. Bund, Birnbaum-Orlicz spaces of functions on groups................. 351

John D. Elwin and Donald R. Short, Branched immersions between 2-manifolds of higher

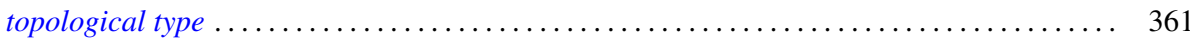

Eric Friedlander, Extension functions for rank 2, torsion free abelian groups . .......... 371

Jon Froemke and Robert Willis Quackenbush, The spectrum of an equational class of

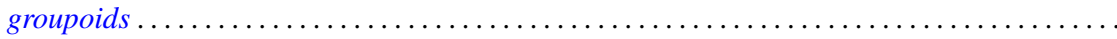

Barry J. Gardner, Radicals of supplementary semilattice sums of associative rings ...........

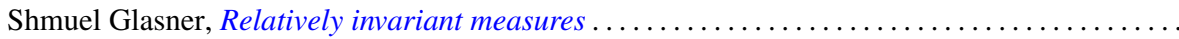

George Rudolph Gordh, Jr. and Sibe Mardesic, Characterizing local connectedness in inverse

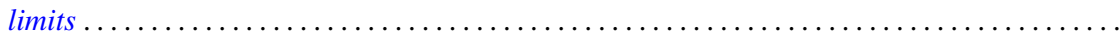

Siegfried Graf, On the existence of strong liftings in second countable topological spaces......

Stanley P. Gudder and D. Strawther, Orthogonally additive and orthogonally increasing

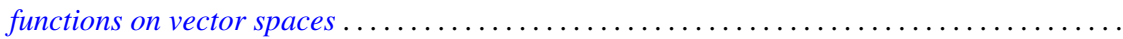

Darald Joe Hartfiel and Carlton James Maxson, A characterization of the maximal monoids and

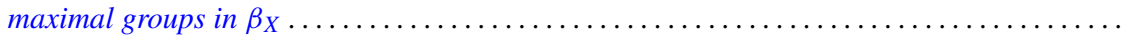

Robert E. Hartwig and S. Brent Morris, The universal flip matrix and the generalized faro-shuffle. .

William Emery Haver, Mappings between ANRs that are fine homotopy equivalences. .

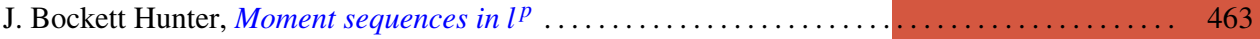

Barbara Jeffcott and William Thomas Spears, Semimodularity in the completion of a poset.... 467

Jerry Alan Johnson, A note on Banach spaces of Lipschitz functions . . . . . . . . . . . . 475

David W. Jonah and Bertram Manuel Schreiber, Transitive affine transformations on

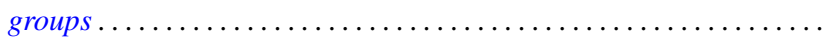

Karsten Juul, Some three-point subset properties connected with Menger's characterization of

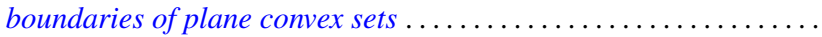

Ronald Brian Kirk, The Haar integral via non-standard analysis . . . . . . . . . . . . . 517

Justin Thomas Lloyd and William Smiley, On the group of permutations with countable

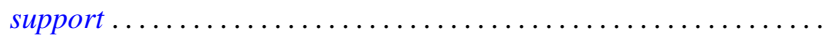

Erwin Lutwak, Dual mixed volumes .................................. 531

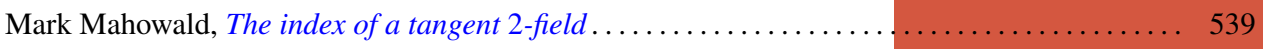

Keith Miller, Logarithmic convexity results for holomorphic semigroups . . . . . . . . . . . . 549

Paul Milnes, Extension of continuous functions on topological semigroups . . . . . . . . . . 553

Kenneth Clayton Pietz, Cauchy transforms and characteristic functions ................ 563

James Ted Rogers Jr., Whitney continua in the hyperspace $C(X) \ldots \ldots \ldots \ldots \ldots \ldots \ldots \ldots .569$

Jean-Marie G. Rolin, The inverse of a continuous additive functional . . . . . . . . . . . . 585

William Henry Ruckle, Absolutely divergent series and isomorphism of subspaces . ........ 605

Rolf Schneider, A measure of convexity for compact sets . ..................... 617

Alan Henry Schoenfeld, Continous measure-preserving maps onto Peano spaces .......... 627

V. Merriline Smith, Strongly superficial elements .......................... 643

Roger P. Ware, A note on quadratic forms over Pythagorean fields . . . . . . . . . . . . . . 651

Roger Allen Wiegand and Sylvia Wiegand, Finitely generated modules over Bezout rings . . . . 655

Martin Ziegler, A counterexample in the theory of definable automorphisms . . . . . . . . . 665 